\title{
MicroRNA-603 functions as an oncogene by suppressing BRCC2 protein translation in osteosarcoma
}

\author{
CHENGBIN MA ${ }^{1}$, CHUAN ZHAN $^{1}$, HONGMOU YUAN $^{2}$, YAN CUI $^{3}$ and ZHIYU ZHANG ${ }^{4 *}$ \\ Departments of ${ }^{1}$ Spinal Surgery, ${ }^{2}$ Traumatology Surgery, ${ }^{3}$ Joint Surgery and ${ }^{4}$ Joint Surgery and Surgical Oncology, \\ Department of Orthopedics, The Fourth Affiliated Hospital of China Medical University, \\ Huanggu District, Shenyang 110032, P.R. China
}

Received October 15, 2015; Accepted November 18, 2015

DOI: $10.3892 /$ or.2016.4718

\begin{abstract}
The present study was conducted to investigate the expression of miR-603 in osteosarcoma cells, and the effect of miR-603 on the biological behavior and expression of breast cancer cell 2 (BRCC2) in osteosarcoma cells. In the present study, qRT-PCR was used to measure the levels of miRNA and mRNA. The results showed that miR-603 was significantly upregulated in human osteosarcoma tissues and cell lines. MTT and colony formation assays were employed to evaluate the role of miR-603 in the regulation of osteosarcoma cell proliferation. The results showed that overexpression of miR-603 promoted the proliferation of MG-63 and U2OS cells. Furthermore, a nude mouse subcutaneous tumor model indicated that miR-603 promoted osteosarcoma growth in vivo. Moreover, miR-603 expression levels were increased in patients with distant metastasis in comparison with levels in patients without distant metastasis. We discovered that BRCC2 may be a target of miR-603. Our results demonstrated that overexpression of miR-603 suppressed BRCC2 protein expression, and an miR-603 inhibitor enhanced BRCC2 protein expression as determined by western blot assay and immunohistochemical analysis. Luciferase reporter assays confirmed that BRCC2 is a direct target of miR-603 in osteosarcoma cells, and the results suggest that miR-603 downregulates BRCC2 expression in osteosarcoma via translational inhibition. Finally, we found that the reduction in BRCC2 expression induced by miR-603 was responsible for the enhanced colony formation and proliferative ability noted in the MG-63 and U2OS cells. In conclusion, miR-603 enhanced osteosarcoma growth by downregulation of BRCC2 expression via translational inhibition.
\end{abstract}

Correspondence to: Professor Zhiyu Zhang, Department of Joint Surgery and Surgical Oncology, Department of Orthopedics, The Fourth Affiliated Hospital of China Medical University, 4 Chongshan Dong Road, Huanggu District, Shenyang 110032, P.R. China

E-mail: zyzhangtg@sina.com

Key words: miR-603, BRCC2, osteosarcoma, oncogene, proliferation

\section{Introduction}

Osteosarcoma is a primary mesenchymal tumor characterized histologically by malignant tumor cells that directly produce osteoid or immature bone. It is the most commonly diagnosed primary malignancy of the bone, particularly among children and adolescents (1). As the disease results in active bone growth and repair (2), it is highly destructive and metastatic. In the last several decades, therapies utilizing surgery and chemical agents have improved the 5-year survival rate of these patients to $\sim 80 \%$ (3). However, the cure rate of patients bearing osteosarcoma is still very poor, and the majority eventually die of metastases (4). Thus, an understanding of the genes and molecules involved in osteosarcoma is particularly critical for developing new therapies and improving patient outcomes.

miR-603 has been reported as a tumor suppressor in thyroid cell tumors (5), laryngeal squamous cell carcinoma (6) and GH adenoma (7). miR-603 has also been previously reported to be overexpressed in pancreatic (8), ovarian (9) and colorectal cancer (10). However, its function in osteosarcoma is not yet known.

Breast cancer cell 2 (BRCC2) is unique among the protooncogenes, being localized to mitochondria and extending cell survival by blocking programmed cell death (11). BRCC2 expression is reported to prevent apoptosis in a wide variety of cell types, including T-lymphocytes, prostate cancer cells and embryonic sensory neurons (12). Previous studies have reported that expression of BRCC2 suppresses cellular proliferation and is associated with less aggressive biological behavior and better prognosis $(13,14)$.

In the present study, we found that miR-603 is overexpressed in osteosarcoma, as compared to normal non-cancer tissue and a normal human osteoblastic cell line. miR-603 significantly promoted osteosarcoma cell growth in vitro and in vivo. We further demonstrated that BRCC2 is a direct target of miR-603. miR-603 appears to be a potential therapeutic target for use in osteosarcoma treatment.

\section{Materials and methods}

Ethical approval. All procedures performed in the studies involving human participants were in accordance with the 
ethical standards of the Institutional and/or National Research Committee and with the 1964 Helsinki Declaration and its later amendments or comparable ethical standards. All applicable international, national and/or institutional guidelines for the care and use of animals were followed.

Tissue samples. From January 2010 to March 2014, a total of 50 primary osteosarcoma and corresponding non-cancerous bone tissue samples were collected from the Fourth Affiliated Hospital of the China Medical University. All specimens were obtained after procuring written informed consent according to a protocol approved by the Institutional Review Board of the Fourth Affiliated Hospital of the China Medical University. Specimens were snap-frozen in liquid nitrogen and stored at $-80^{\circ} \mathrm{C}$ for quantitative real-time reverse-transcriptase polymerase chain reaction (qRT-PCR) analysis. All of the 50 patients received follow-up. The median follow-up was 60 months (range 4-62 months).

Cell lines and maintenance. Human osteosarcoma cell lines (MG-63, Saos-2, U2OS and 143B) and human normal osteoblastic cell line hFOB1.19 were obtained from the Type Culture Collection of the Chinese Academy of Sciences (Shanghai, China). The cell lines mentioned above were cultured in Dulbecco's modified Eagle's medium (DMEM) (Invitrogen, USA) supplemented with $10 \%(\mathrm{w} / \mathrm{v})$ fetal bovine serum (FBS) and incubated at $37^{\circ} \mathrm{C}$ with $5 \%(\mathrm{v} / \mathrm{v}) \mathrm{CO}_{2}$.

RNA extraction and quantitative real-time PCR. Total RNAs were extracted from the cultured human tissue specimens and cells using TRIzol reagent (Invitrogen) according to the manufacturer's instructions. For the detection of mature miR-603 and relative mRNA, RNA was reverse transcribed using a TaqMan reverse transcription kit (Applied Biosystems, Foster City, CA, USA). The reaction was incubated at $94^{\circ} \mathrm{C}$ for $4 \mathrm{~min}$ followed by 35 cycles of $20 \mathrm{sec}$ at $94^{\circ} \mathrm{C}, 30 \mathrm{sec}$ at $60^{\circ} \mathrm{C}$ and $30 \mathrm{sec}$ at $72^{\circ} \mathrm{C}$. All PCRs were carried out in triplicate on an ABI 7500 Real-Time PCR system (Applied Biosystems), and miRNA and mRNA expression was normalized to U6 snRNA and GAPDH, respectively, using the $2^{-\Delta \Delta C t}$ method, and at least 3 independent experiments were performed to generate each data set.

Tumor formation in nude mice. Nude mice (4-weeks old, 18.0-22.0 $\mathrm{g}$ in weight) were subcutaneously injected with $2 \times 10^{6}$ cells transfected with either miR-603 mimics or its inhibitor. Every 2 days post-inoculation, the length and width of the implanted tumors were measured with a Vernier caliper. After 3 weeks, the mice were sacrificed to measure tumor weights. The mice were manipulated and housed according to protocols approved by the Local Medical Experimental Animal Care Commission.

Transfection. miRNA-603 mimics and its inhibitor were purchased from GenePharma Co. Ltd. (Shanghai, China). For transfection, MG-63 and U2OS cells were grown to 90\% confluency, and transfected with miR-603 mimics or its inhibitor using Lipofectamine 2000 (Invitrogen) by incubation in Opti-Mem I media for $4 \mathrm{~h}$ according to the manufacturer's protocols. Cells were harvested 48 or $72 \mathrm{~h}$ post-transfection.
The transfection efficiency was confirmed by quantitative real-time PCR analysis. For detecting BRCC2, the cells were transfected again with BRCC2 or its silence gene.

Luciferase reporter assays. Cells cultured into 6-well plates were transfected with $0.05 \mu \mathrm{g}$ of the pRL-TK vector (Promega, Madison, WI, USA) containing Renilla luciferase along with $30 \mathrm{nM}$ miR-603 mimics or inhibitor. Cells cultured for $24 \mathrm{~h}$ were then transfected with BRCC2-wild-type (WT) or BRCC2-mutant reporter plasmid containing firefly luciferase using Lipofectamine 2000. Luciferase activity was measured using a dual luciferase assay system (Promega) 48 h posttransfection. Renilla activity was normalized to firefly activity to control for transfection efficiency.

MTT assay. The transfected cells were seeded into 96-well plates at a density of $3 \times 10^{3}$ cells/well. MTT solution $(20 \mu \mathrm{l}$ of $5 \mathrm{mg} / \mathrm{ml} \mathrm{MTT}$ ) was added to each well (for a total volume of $250 \mu \mathrm{l}$ ), and the plates were incubated for $4 \mathrm{~h}$ at $37^{\circ} \mathrm{C}$. Following the removal of the culture medium, the remaining crystals were dissolved in dimethylsulfoxide (DMSO), and the absorbance was measured at $570 \mathrm{~nm}$ using a microplate reader. Cell proliferation was assessed daily for 4 consecutive days.

Colony formation assay. Different cells were seeded in triplicate at 500 cells $/ 6-\mathrm{cm}$ dishes in complete medium. The cells were then cultured in DMEM containing 10\% FBS and DMEM was changed every 2 days. After incubation for 15 days, the cells were fixed with methanol and stained with $0.1 \%$ crystal violet. Visible colonies were manually counted. Triplicate wells were measured for each group.

Immunohistochemistry. All steps were carried out at room temperature as previously described (15). Cells transfected with miR-603 mimics or its inhibitor were plated on poly-Dlysine-coated glass coverslips $72 \mathrm{~h}$ post-transfection and were fixed in 4\% (w/v) paraformaldehyde (Electron Microscopy Sciences) for $1 \mathrm{~h}$, washed in phosphate-buffered saline (PBS) (Invitrogen) (6 times, 5 min each), incubated for $1 \mathrm{~h}$ in blocking buffer (PBS, $0.8 \%$ Triton X-100, 10\% normal goat serum), and then incubated overnight in blocking buffer with anti-BRCC2 (1:100) antibodies. The cells were washed in PBS (6 times, $5 \mathrm{~min}$ each) before the addition of goat anti-mouse secondary antibodies (Invitrogen, Molecular Probes) at 1:250 for $60 \mathrm{~min}$ followed by washing in PBS (6 times, 5 min each). Finally, the slides were incubated with 3,3'-diaminobenzidine (BioGenex Laboratories, San Ramon, CA, USA) and color development was closely monitored under a microscope. The slides were counterstained with hematoxylin.

Western blotting. Western blotting was used to detect the expression of BRCC2 at the protein level as previously described (15). Anti- $\beta$-actin (Abcam) was used as the protein-loading control. The protein complex was detected with enhanced chemiluminescence reagents. Digital images were visualized using the electrochemiluminescence detection system (Invitrogen).

RNA interference for BRCC2. One siRNA lentivirus against BRCC2 and a non-targeting siRNA (both from Sigma-Aldrich) were transfected into U2OS-anti-miR-603 cells in 48-well 
A

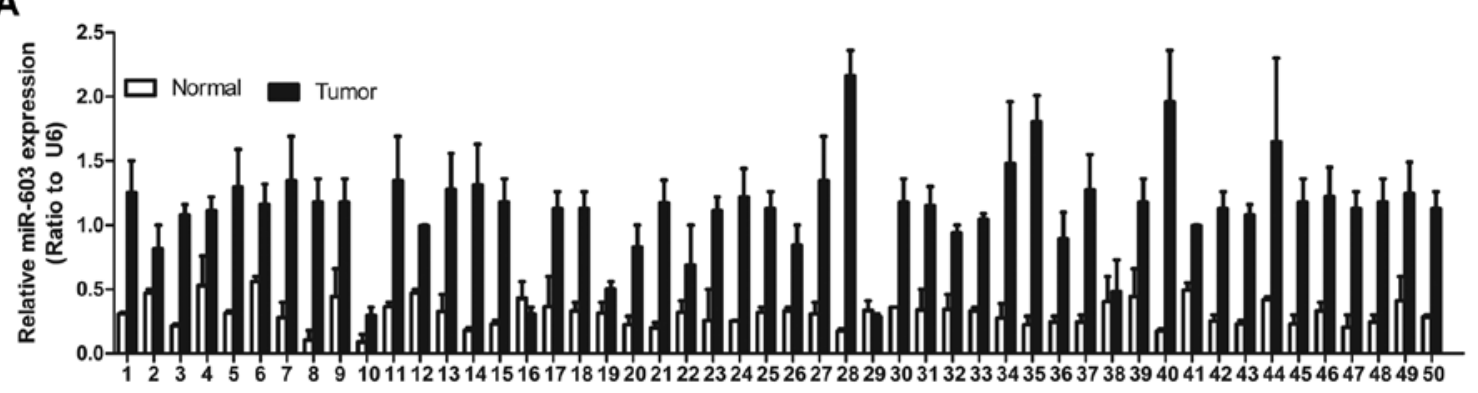

B

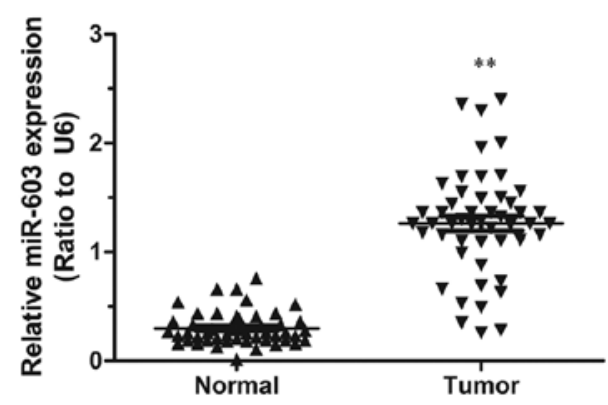

C

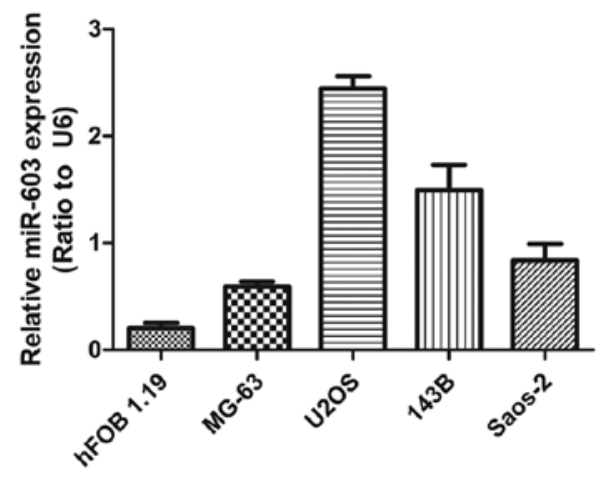

plates according to the manufacturer's instructions. The multiplicity of infection (MOI; number of transducing lentiviral particles/cell) was 5. Puromycin selection was performed at a concentration of $0.5 \mu \mathrm{g} / \mathrm{ml}$ for 10 days.

Statistical analysis. All data are presented as the mean \pm standard error of the mean (SEM). Statistical significance was determined using t-test or analysis of variance (ANOVA) using the SPSS 18.0 program. $\mathrm{p}<0.05$ was considered to indicate a statistically significant difference.

\section{Results}

miR-603 is upregulated in human osteosarcoma carcinoma tissues and cell lines. To explore the expression level of miR-603 in human osteosarcoma carcinoma development, miR-603 expression was assessed in 50 paired osteosarcoma carcinoma and adjacent non-tumor tissues. According to the qRT-PCR analysis, miR-603 was strongly upregulated in the 50 tumor tissues by 1.5 - to 6 -fold when compared with that in the matched non-tumor tissues (Fig. 1A and B). We next examined miR-6-3 expression levels in a panel of 4 widely used human osteosarcoma cell lines in comparison to levels in the non-malignant cell line hFOB 1.19. Correspondingly,
D

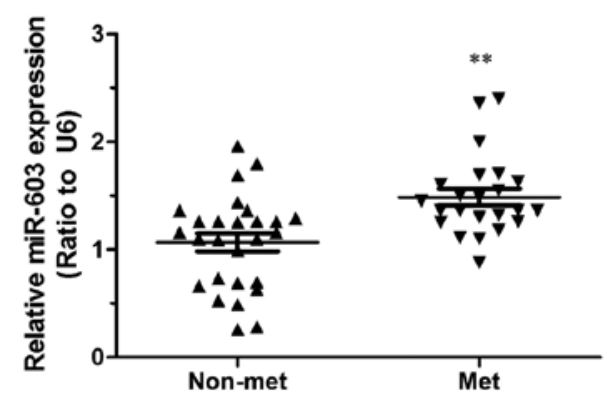

Figure 1. Expression of miR-603 is elevated in osteosarcoma tissues and cell lines. (A) miR-603 expression levels are measure by qPCR in osteosarcoma tissues and normal tissues. (B) miR-603 expression levels are significantly elevated in osteosarcoma tissues in comparison to normal tissues. (C) Expression profile of miR-603 in osteosarcoma cell lines. miR-603 RNA levels relative to normal osteoblastic cell line hFOB 1.19 were determined by qPCR. (D) miR-603 expression levels were significantly increased in patients of metastasis in comparison to non-metastasis. Data are presented as means \pm standard deviation. ${ }^{* *} \mathrm{p}<0.01$, unpaired two-tailed Student's t-test. The experiments were performed in triplicate.

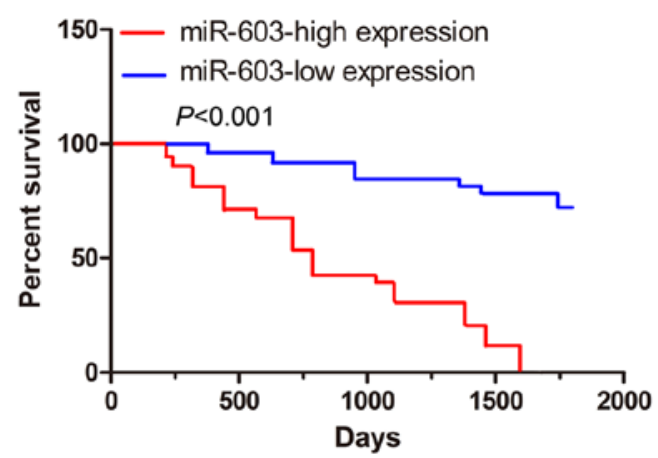

Figure 2. Elevated expression of miR-603 is associated with reduced osteosarcoma patient survival.

miR-603 expression levels were consistently increased in the osteosarcoma cell lines (Fig. 1C). To investigate the clinical impact of elevated miR-603 expression in osteosarcoma, we assessed the association between miR-603 expression levels and clinical outcome. miR-603 expression levels were statistically increased in patients of stage III-IV in comparison to the stage I-II (Fig. 1D). Furthermore, in order to determine the prognostic impact of miR-603 expression in osteosarcoma, we categorized osteosarcoma patients into two groups based 
A

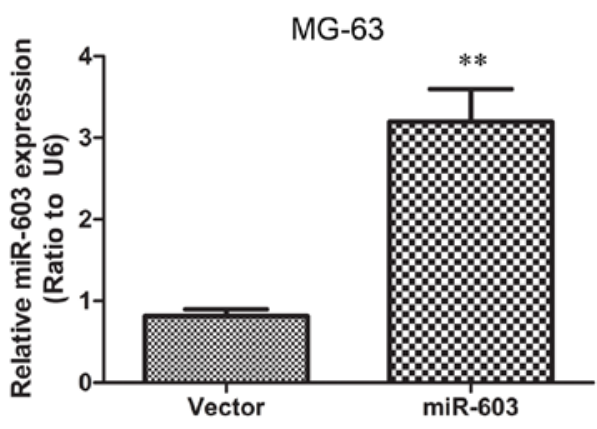

C

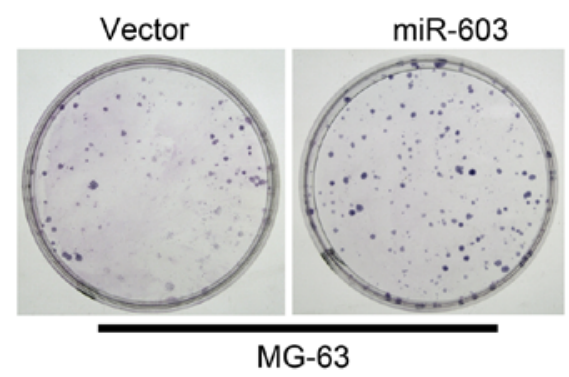

B

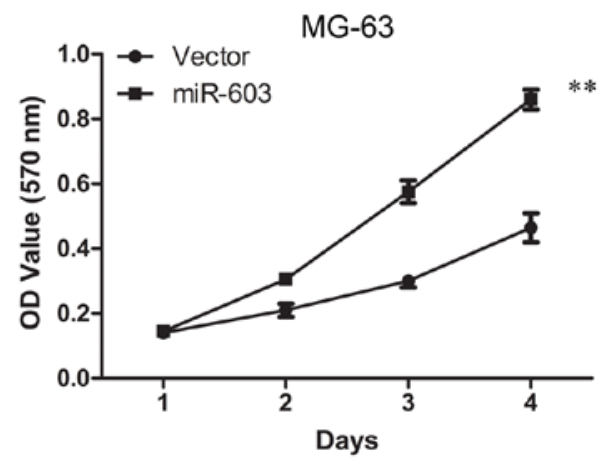

MG-63

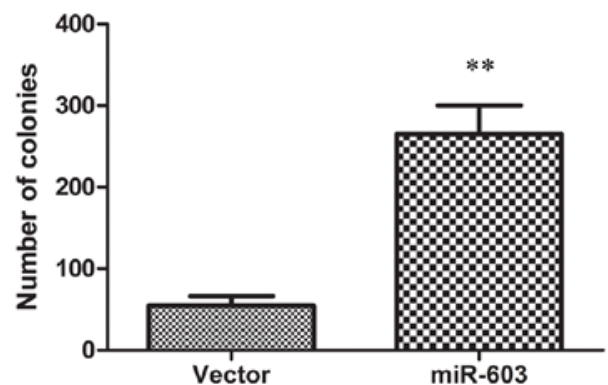

Figure 3. Overexpression of miR-603 enhances tumorigenesis in vitro and in vivo. (A) Establishment of MG-63-overexpressing miR-603 cells. The results were analyzed by qRT-PCR. (B) Proliferation of MG-63-miR-603 cells was significantly accelerated compared to the normal MG-63 control cells as determined by MTT assay. (C) Representative stained colonies are displayed in the left panel. The number of colonies is shown in the right panel. ${ }^{* *}$ p $<0.01$, unpaired two-tailed Student's t-test. The experiments were performed in triplicate.

on miR-603 expression levels. Patients with tumors displaying high miR-603 expression levels had a significantly reduced rate of survival compared to those with low miR-603 (Fig. 2).

Overexpression of miR-603 in MG-63 cells enhances tumorigenesis in vitro and in vivo. To evaluate a possible involvement of miR-603 in osteosarcoma tumorigenesis, we examined its expression level in human osteosarcoma. We established MG-63 cells overexpressing miR-603 by introducing miR-603 mimics using lentiviral vectors. The miR-603 expression levels were tested using qRT-PCR, and the results showed that the miR-603 group expressed higher miR-603 levels as compared with the vector group (Fig. 3A). We next tested the hypothesis that overexpression of miR-603 in MG-63 cells promotes cell growth and colony formation ability. We performed MTT and colony formation assays. The MTT assay showed that high expression of miR-603 resulted in more rapid proliferation compared with the vector group (Fig. 3B). Furthermore, transfection with miR-603 mimics resulted in significantly promoted colony formation under serum starvation (Fig. 3C). The two results indicated that miR-603 is potentially oncogenic. The miR-603-mediated tumorigenic effects were confirmed in a nude mouse subcutaneous tumor model. The results demonstrated that cells in the miR-603 group formed progressively growing solid tumors. By contrast, cells in the vector group produced much smaller tumors (Fig. 4A). Finally, as shown in Fig. 4A and B, overexpression of miR-603 promoted tumor growth in vivo. All of these findings above demonstrated that miR-603 induces a more aggressive phenotype of osteosarcoma carcinoma.
A

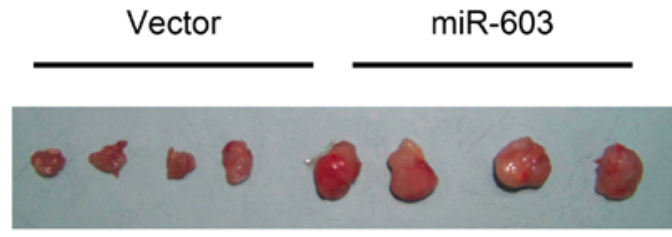

MG-63

\section{B}

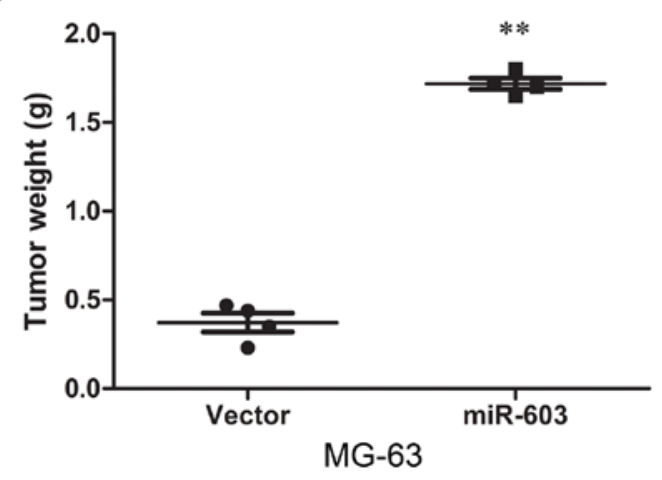

Figure 4. Overexpression of miR-603 promotes tumor growth in vivo. (A) Subcutaneous tumors in 4 nude mice are displayed. (B) The weights of tumors are shown in the graph. ${ }^{* *} \mathrm{p}<0.01$, unpaired two-tailed Student's t-test. The experiments were performed in triplicate.

Inhibition of miR-603 in U2OS cells reduces tumorigenesis in vitro and in vivo. We suppressed miR-603 expression in U2OS cells by introducing anti-miR-603 mimics using lentiviral vectors. The suppression of miR-603 expression 
A

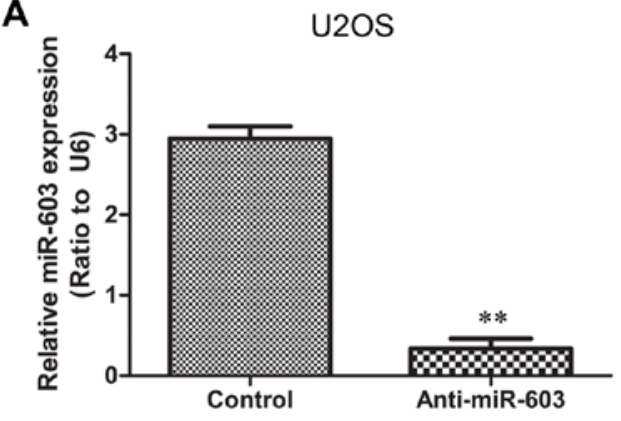

C

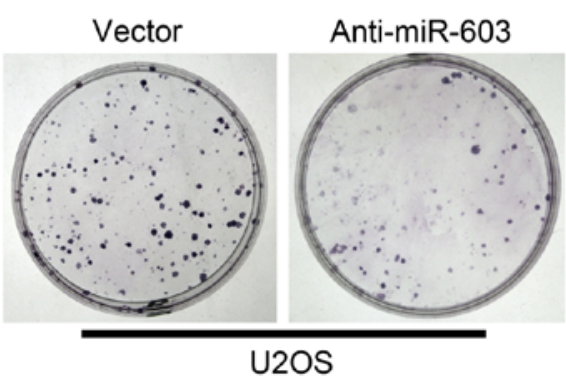

B
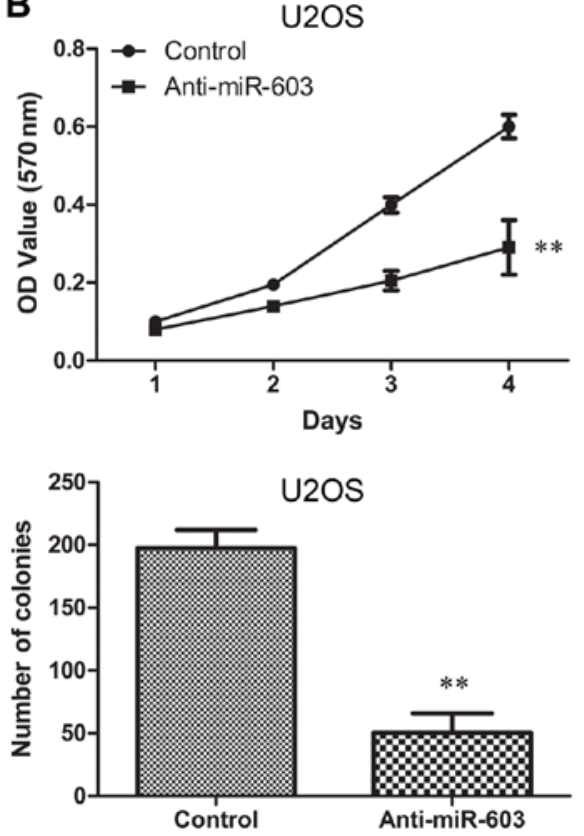

Figure 5. Downregulation of miR-603 reduces tumorigenesis in vitro and in vivo. (A) Establishment of the downregulation of miR-603 in U2OS cells. The results were analyzed by qRT-PCR. (B) The proliferation of U2OS-anti-miR-603 cells was significantly reduced compared to the normal U2OS control cells as determined by MTT assay. (C) Colony formation assay. Representative stained colonies are displayed in the left panel. ${ }^{* *}$ p $<0.01$, unpaired two-tailed Student's t-test. The experiments were performed in triplicate.

was confirmed using qRT-PCR (Fig. 5A). To corroborate this hypothesis, we investigated whether downregulation of miR-603 in U2OS cells reduces cell growth and colony formation ability. MTT and colony formation assays were also carried out. The proliferation curve showed that cells in the anti-miR-603 group exhibited significant decline in proliferation when compared to cells in the vector group (Fig. 5B). Furthermore, transfection with anti-miR-603 mimics obviously restrained colony formation under serum starvation (Fig. 5C). Animal studies were conducted to further evaluate the effect of anti-miR-603 on osteosarcoma tumor growth in a nude mouse subcutaneous tumor model. The results demonstrated that cells in the vector group formed progressively growing solid tumors. By contrast, in the anti-miR-603 group much smaller tumors were formed (Fig. 6A). Finally, the mean weight of tumors in the control group was significantly higher than that in the anti-miR-603 group (Fig. 6B).

miR-603 reduces the protein levels of BRCC2 by inhibiting translation. In order to explore the mechanisms by which miR-603 promotes osteosarcoma tumorigenesis, in silico prediction models were employed to search for genes regulated by miR-603. We chose to investigate signal transducer and activator of BRCC2 as a possible target of miR-603 since it is a known tumor-suppressor gene that has been previously reported to be a direct target of miR-603. To confirm that BRCC2 is a target of miR-603, the RNA levels of BRCC2 were assessed in MG-63 cells overexpressing miR-603 (Fig. 7C) and U2OS cells with silenced miR-603 (Fig. 7D). Correlation between miR-603 and BRCC2 expression was assessed by western blot analysis (Fig. 8A) and the immunohistochemical analysis in vivo (Fig. 8B and C). We found that BRCC2 was downregulated in the miR-603-overexpressing cells, whereas

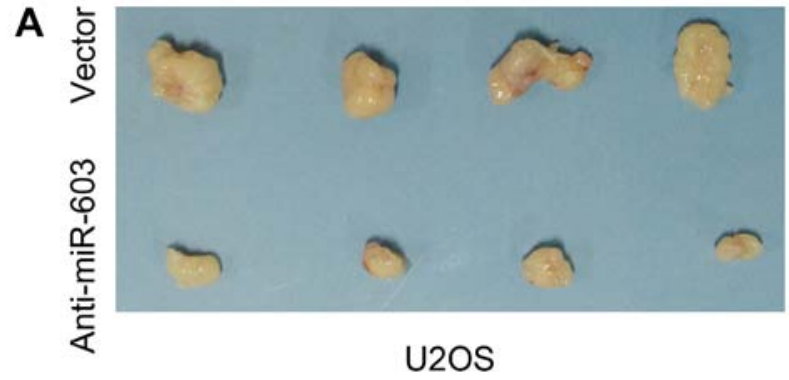

B

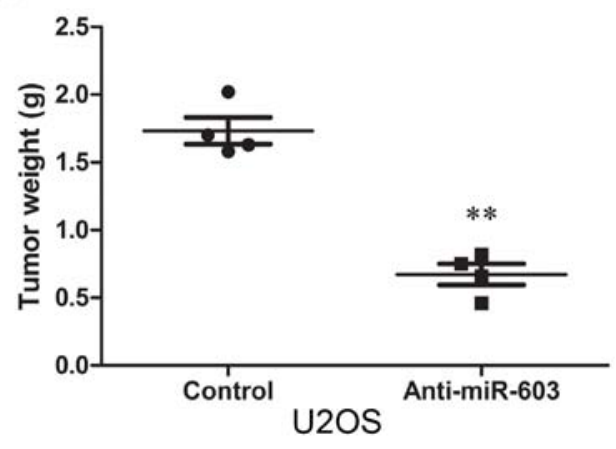

Figure 6. Anti-miR-603 suppresses tumor growth in an in vivo model. (A) Subcutaneous tumors in 4 nude mice are displayed. (B) Weights of tumors are shown in the graph. ${ }^{* *} \mathrm{p}<0.01$, paired two-tailed Student's t-test. The experiments were performed in triplicate.

BRCC2 was increased by the miR-603-specific inhibitor compared with that observed in the vector cells. We also repeated the luciferase assay to confirm BRCC2 as a target of miR-603 in osteosarcoma cells, and assessed whether 
A

$\begin{array}{cc}\text { BRCC2-3'UtR } & \text { 5' CUAGgCCGAUUUAAUgAagCCC 3' } \\ \text { miR-603 } & \text { CACACACUGCAAUUACUUUUGC }\end{array}$
C

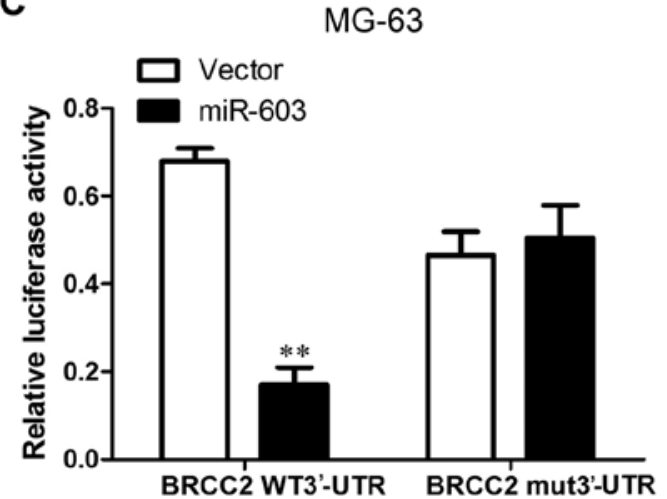

B

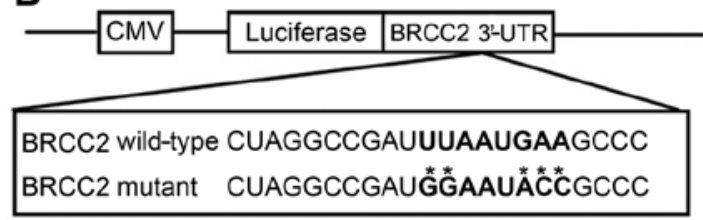

D

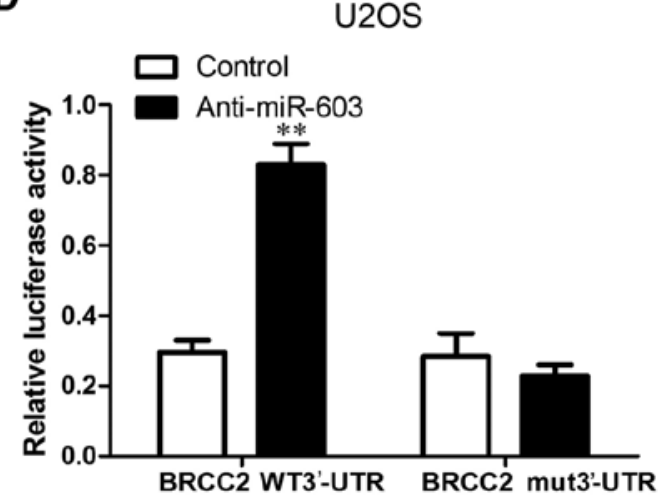

Figure 7. miR-603 reduces the protein levels of BRCC2 by inhibiting translation. (A) Potential binding site for miR-603 in the 3 '-UTR of BRCC2 mRNA. (B) BRCC2 3'-UTR possesses a mutation in the putative miR-603 binding site. (C) The luciferase activity after transfection in the MG-63 cells of the indicated 3'-UTR-driven reporter constructs. Reporter plasmids containing no oligonucleotides as a control, the wild-type 3'-UTR region of BRCC2 as a wild-type and the mutant 3 '-UTR region as a mutant. ${ }^{* *} p<0.01$, unpaired two-tailed Student's t-test. (D) Luciferase activity after transfection in U2OS cells of the indicated 3'-UTR-driven reporter constructs. Reporter plasmids containing no oligonucleotides as a control, the wild-type 3'-UTR region of BRCC2 as a wild-type and the mutant 3 '-UTR region as a mutant. ${ }^{* *} \mathrm{p}<0.01$, unpaired two-tailed Student's t-test. The experiments were performed in triplicate.

miR-603 inhibits the translation of BRCC2. One potential binding site for miR-603 was located on the 3'-UTR of BRCC2 mRNA (Fig. 7A and B). To further validate whether a miR-603 binding site in the BRCC2 3'-UTR mediated this suppression, we inserted the BRCC2 3'-UTR transcript or a mutated version into the luciferase system. The detection of luciferase activity showed that the activity of luciferase combined with wild-type BRCC2 3'-UTR was decreased in the MG-63-miR-603 cells and increased in the U2OS-anti-miR-603 cells, whereas the luciferase activity was not altered by the vector when BRCC2 3'-UTR possessing a mutation in the putative miR-603 binding site. As no significant differences in the BRCC2 mRNA levels were observed between the MG-63 vector as well as U2OS controls, miR-603 did not appear to degrade BRCC2 mRNA (Fig. 7C and D). These results together suggest that miR-603 downregulates BRCC2 expression in osteosarcoma via translational inhibition.

BRCC2 is responsible for the colony-forming and proliferative ability of MG-63 and U2OS cells. In order to investigate the correlation between BRCC2 expression and colony-forming and proliferativen ability of the osteosarcoma cells, we constructed MG-63-miR-603 cells transfected with BRCC2 and U2OS-anti-miR-603 cells transfected with specific siRNAs against BRCC2. The expression of BRCC2 at the protein level was analyzed by western blotting. The results confirmed that BRCC2 expression was significantly upregulated in the MG-63-miR-603 cells (Fig. 9A) and silenced in U2OS-anti-miR-603 cells (Fig. 10A). Then, the treated cells were evaluated for tumorigenesis using MTT and colony formation assays. As expected, overexpression of BRCC2 strongly suppressed cell proliferation compared

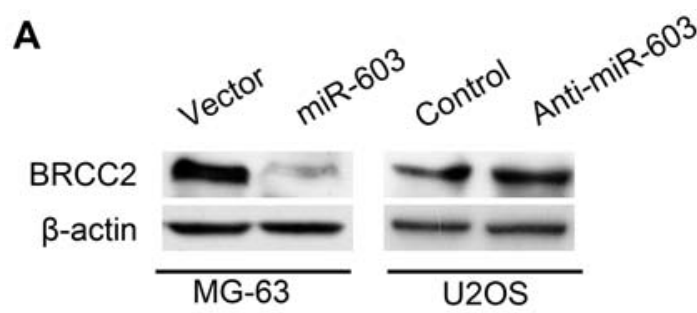

B

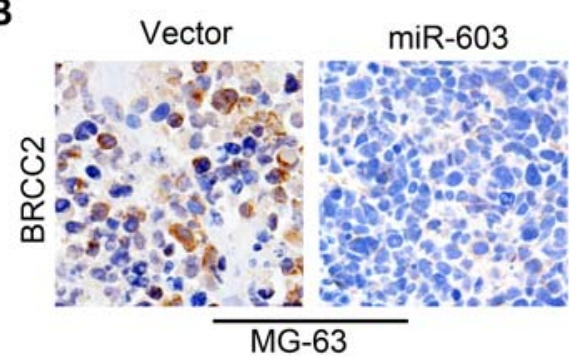

C

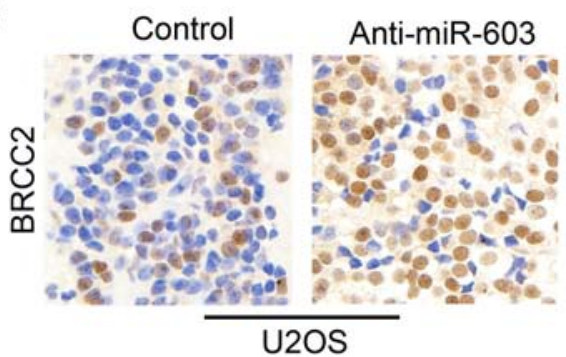

Figure 8. BRCC2 is a potential target of miR-603. (A) Western blot analysis was performed to detect the expression of BRCC 2 and internal control $\beta$-actin in the MG-63 and U2OS cells following transfections. (B and C) Correlation between the miR-603 expression and results of the immunohistochemical analysis of BRCC2 in vivo. Representative results are shown in micrographs; magnification, $\mathrm{x} 100$. All experiments were performed in triplicate. 
A

\section{B}
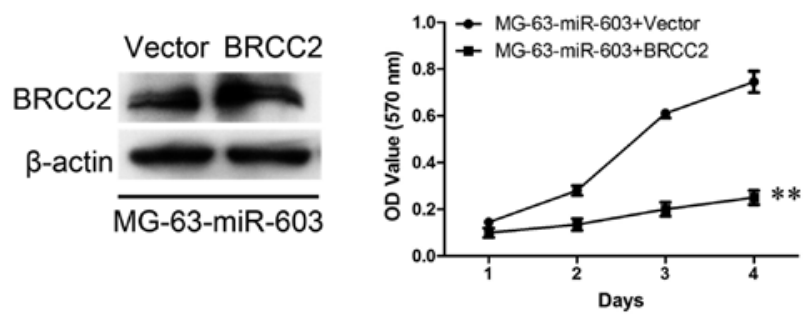

C

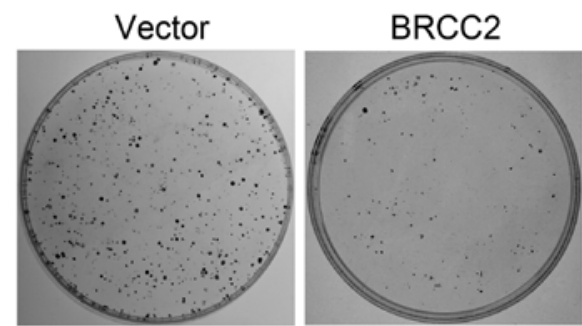

MG-63-miR-603

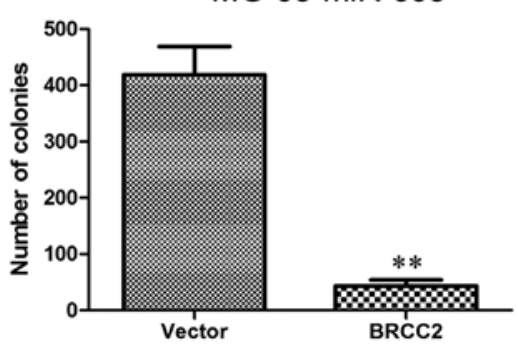

Figure 9. BRCC2 expression suppresses the colony formation and proliferation phenotype of MG-63 cells. (A) MG-63-miR-603 cells were transfected with BRCC2, and the results of immunoblotting for BRCC2 and $\beta$-tubulin are shown. (B) BRCC2-transfected MG-63-miR-603 cells showed a significant reduction in proliferation in comparison to the control cells (C) MG-63-miR-603 cells transfected with BRCC2 showed reduced colony formation. ${ }^{* *}$ p $<0.01$, unpaired two-tailed Student's t-test. The experiments were performed in triplicate.

to the vector cells (Fig. 9B) and clearly decreased colony formation ability (Fig. 9C). Inhibition of BRCC2 expression strongly promoted cell proliferation compared to the control cells (Fig. 10B) and increased colony formation (Fig. 10C). Collectively, these results indicate that reduced BRCC2 expression induced by miR-603 is responsible for colony-forming and proliferation ability of the MG-63 and U2OS cells.

\section{Discussion}

miRNAs are a type of small-molecule non-coding RNAs, which play an important role in the regulation of post-transcriptional gene expression levels. The functions of miRNAs vary in various clinical diseases and may regulate all aspects of bioactivities, including differentiation and development, metabolism, proliferation, apoptotic cell death, viral infection and tumorigenesis (16). Abnormal cellular development, as occurs in cancer, has also been associated with miRNAs (17). The miRNAs with increased expression levels in tumors may function as oncogenes by negatively regulating tumorsuppressor genes. In contrast, the miRNAs downregulated in cancer may function as tumor suppressors and inhibit cancer development by downregulating responding oncogenes (18).
A

B
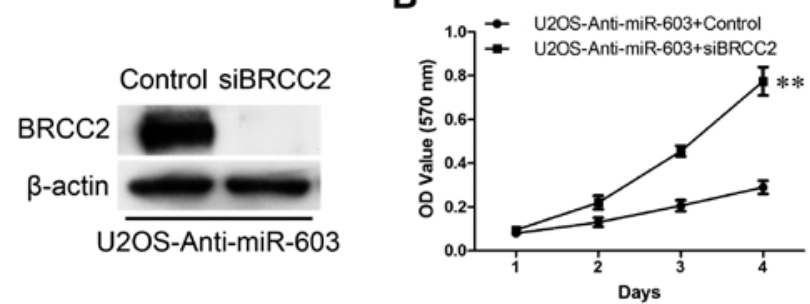

C

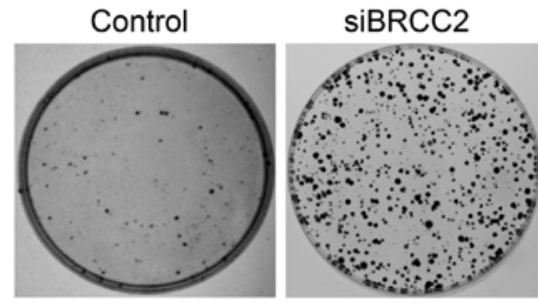

U2OS-Anti-miR-603

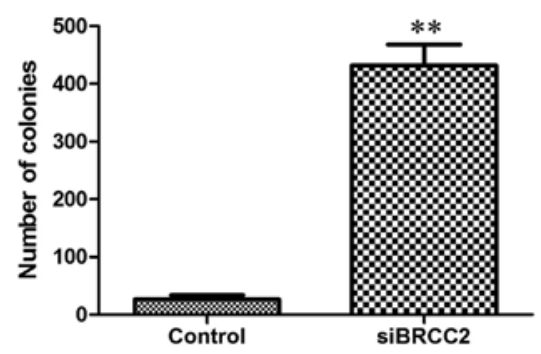

Figure 10. Reduced BRCC2 expression enhances the colony formation and proliferation phenotype of U2OS cells. (A) Knockdown of BRCC2 in U2OS-anti-miR-603 cells. The results of immunoblotting for BRCC2 and $\beta$-tubulin are shown. (B) Knockdown of BRCC2 in U2OS-anti-miR-603 cells enhanced the proliferative ability of the U2OS-anti-miR-603 cells. (C) Knockdown of BRCC2 in U2OS-anti-miR-603 cells increased colony formation in the U2OS-anti-miR-603 cells. ${ }^{* *} \mathrm{p}<0.01$, unpaired two-tailed Student's t-test. The experiments were performed in triplicate.

To summarize, miRNAs participate in all stages of tumor development.

Osteosarcoma is one of the most common tumors that threats human life. The incidence of osteosarcoma accounts for $20 \%$ of all primary malignant bone tumors, behind multiple myeloma. Typical osteosarcoma is a highly malignant intramedullary tumor, accounting for $85 \%$ of all bone sarcoma (19). Study of the relationships between miRNAs and osteosarcoma may provide a new direction for the diagnosis and treatment of osteosarcoma. In previous studies, miR-143, miR-145, miR-195, miR-133a, miR-218 and miR-34a were reported to inhibit osteosarcoma cell proliferation and to suppress tumorigenicity (20-25); miR-221, miR-214, miR-128, miR-181, miR-33a and miR-27a were reported to induce osteosarcoma cell survival and promote cell proliferation (26-31).

It appears that miR-603 functions differentially in various types of tumors. Our results found that it acts as an oncogene by suppressing BRCC2. BRCC2 expression is reported to prevent apoptosis in a wide variety of cell types and as a target of miRNAs. Suppression or induction of BRCC2 affects tumorigenesis. miR-195, miR-24-2 and miR-365-2 were reported as negative regulators of $\mathrm{BRCC} 2$ through direct binding to their respective binding sites in the 3'-UTR of the human BRCC2 
gene (32). While, miR-15 and miR-16 induce apoptosis by targeting BRCC2 (33).

The definition of osteosarcoma is deceptively straightforward. It is a malignant tumor of connective tissue origin within which tumor cells produce bone or osteoid (34). Our results revealed that miR-603 enhanced tumor growth by downregulation of $\mathrm{BRCC} 2$ expression via translational inhibition in osteosarcoma cell lines and clinical samples. Increased miR-603 expression was associated with aggressive clinicopathological features and poor prognosis. The suppression of miR-603 exhibited an antitumor effect both in vitro and in vivo. Our findings demonstrate that miR-603 may be a potential novel target for the gene therapy of osteosarcoma.

In summary, the present study showed that miR-603 is significantly upregulated in osteosarcoma, meanwhile it is related with poor patient overall survival. This demonstrates that miR-603 has powerful oncogenic, proliferative and invasive regulatory effects that are mediated by BRCC 2 . The study indicates that miR-603 acts as an oncogene and is a promising therapeutic target in osteosarcoma.

\section{References}

1. Mirabello L, Troisi RJ and Savage SA: Osteosarcoma incidence and survival rates from 1973 to 2004: Data from the Surveillance, Epidemiology, and End Results Program. Cancer 115: 1531-1543, 2009.

2. Geller DS and Gorlick R: Osteosarcoma: A review of diagnosis, management, and treatment strategies. Clin Adv Hematol Oncol 8: 705-718, 2010.

3. Zhang Y, Zhang L, Zhang G, Li S, Duan J, Cheng J, Ding G, Zhou $\mathrm{C}$, Zhang J, Luo P, et al: Osteosarcoma metastasis: Prospective role of ezrin. Tumour Biol 35: 5055-5059, 2014.

4. O'Farrill JS and Gordon N: Autophagy in osteosarcoma. Adv Exp Med Biol 804: 147-160, 2014.

5. Mussnich P, D'Angelo D, Leone V, Croce CM and Fusco A: The High Mobility Group A proteins contribute to thyroid cell transformation by regulating miR-603 and miR-10b expression. Mol Oncol 7: 531-542, 2013.

6. Ayaz L, Görür A, Yaroğlu HY, Ozcan C and Tamer L: Differential expression of microRNAs in plasma of patients with laryngeal squamous cell carcinoma: Potential early-detection markers for laryngeal squamous cell carcinoma. J Cancer Res Clin Oncol 139: 1499-1506, 2013.

7. D'Angelo D, Palmieri D, Mussnich P, Roche M, Wierinckx A, Raverot G, Fedele M, Croce CM, Trouillas J and Fusco A: Altered microRNA expression profile in human pituitary GH adenomas: Down-regulation of miRNA targeting HMGA1, HMGA2, and E2F1. J Clin Endocrinol Metab 97: E1128-E1138, 2012.

8. Yu J, Li A, Hong SM, Hruban RH and Goggins M: MicroRNA alterations of pancreatic intraepithelial neoplasias. Clin Cancer Res 18: 981-992, 2012.

9. Taylor DD and Gercel-Taylor C: MicroRNA signatures of tumorderived exosomes as diagnostic biomarkers of ovarian cancer. Gynecol Oncol 110: 13-21, 2008.

10. Wang FJ, Ding Y, Mao YY, Jing FY, Zhang ZY, Jiang LF, Guo JF Sun XJ, Jin MJ and Chen K: Associations between hsa-miR-603 polymorphism, lifestyle-related factors and colorectal cancer risk. Cancer Biomark 14: 225-231, 2014.

11. Zutter M, Hockenbery D, Silverman GA and Korsmeyer SJ: Immunolocalization of the $\mathrm{Bcl}-2$ protein within hematopoietic neoplasms. Blood 78: 1062-1068, 1991.

12. Pietenpol JA, Papadopoulos N, Markowitz S, Willson JK, Kinzler KW and Vogelstein B: Paradoxical inhibition of solid tumor cell growth by bcl2. Cancer Res 54: 3714-3717, 1994.

13. EI-Emshaty HM, Saad EA, Toson EA, Abdel Malak CA and Gadelhak NA: Apoptosis and cell proliferation: Correlation with BCL-2 and P53 oncoprotein expression in human hepatocellular carcinoma. Hepatogastroenterology 61: 1393-1401, 2014.
14. Garewal J, Garewal R and Sircar K: Expression of Bcl-2 and MIB-1 markers in oral squamous cell carcinoma (OSCC) - A comparative study. J Clin Diagn Res 8: QC01-QC04, 2014.

15. Wang Y, Wen M, Kwon Y, Xu Y, Liu Y, Zhang P, He X, Wang Q, Huang Y, Jen KY, Labarge MA, et al: CUL4A induces epithelialmesenchymal transition and promotes cancer metastasis by regulating ZEB1 expression. Cancer Res 74: 520-531, 2014.

16. Huang Y, Shen XJ, Zou Q, Wang SP, Tang SM and Zhang GZ: Biological functions of microRNAs: A review. J Physiol Biochem 67: 129-139, 2011.

17. Shenouda SK and Alahari SK: MicroRNA function in cancer: Oncogene or a tumor suppressor? Cancer Metastasis Rev 28: 369-378, 2009

18. Zhang B, Pan X, Cobb GP and Anderson TA: microRNAs as oncogenes and tumor suppressors. Dev Biol 302: 1-12, 2007.

19. Unni KK (ed): Chordoma. In: Dahlin's Bone Tumors: General Aspects and Data on 11,087 cases. 5th edition. Lippincott-Raven, New York, NY, pp291-305, 1996.

20. Osaki M, Takeshita F, Sugimoto Y, Kosaka N, Yamamoto Y, Yoshioka Y, Kobayashi E, Yamada T, Kawai A, Inoue T, et al: MicroRNA-143 regulates human osteosarcoma metastasis by regulating matrix metalloprotease-13 expression. Mol Ther 19: 1123-1130, 2011

21. Fan L, Wu Q, Xing X, Wei Y and Shao Z: MicroRNA-145 targets vascular endothelial growth factor and inhibits invasion and metastasis of osteosarcoma cells. Acta Biochim Biophys Sin 44: 407-414, 2012.

22. Mao JH, Zhou RP, Peng AF, Liu ZL, Huang SH, Long XH and Shu Y: microRNA-195 suppresses osteosarcoma cell invasion and migration in vitro by targeting FASN. Oncol Lett 4: 1125-1129, 2012.

23. Ji F, Zhang H, Wang Y, Li M, Xu W, Kang Y, Wang Z, Wang Z, Cheng P, Tong D, et al: MicroRNA-133a, downregulated in osteosarcoma, suppresses proliferation and promotes apoptosis by targeting Bcl-xL and Mcl-1. Bone 56: 220-226, 2013.

24. Jin J, Cai L, Liu ZM and Zhou XS: miRNA-218 inhibits osteosarcoma cell migration and invasion by down-regulating of TIAM1, MMP2 and MMP9. Asian Pac J Cancer Prev 14: 3681-3684, 2013.

25. Yan K, Gao J, Yang T, Ma Q, Qiu X, Fan Q and Ma B: MicroRNA-34a inhibits the proliferation and metastasis of osteosarcoma cells both in vitro and in vivo. PLoS One 7: e33778, 2012.

26. Zhao G, Cai C, Yang T, Qiu X, Liao B, Li W, Ji Z, Zhao J, Zhao H, Guo M, et al: MicroRNA-221 induces cell survival and cisplatin resistance through PI3K/Akt pathway in human osteosarcoma. PLoS One 8: e53906, 2013.

27. Wang Z, Cai H, Lin L, Tang M and Cai H: Upregulated expression of microRNA-214 is linked to tumor progression and adverse prognosis in pediatric osteosarcoma. Pediatr Blood Cancer 61: 206-210, 2014.

28. Shen L, Chen XD and Zhang YH: MicroRNA-128 promotes proliferation in osteosarcoma cells by downregulating PTEN. Tumour Biol 35: 2069-2074, 2014.

29. Jianwei Z, Fan L, Xiancheng L, Enzhong B, Shuai L and Can L: MicroRNA 181a improves proliferation and invasion, suppresses apoptosis of osteosarcoma cell. Tumour Biol 34: 3331-3337, 2013.

30. Zhou Y, Huang Z, Wu S, Zang X, Liu M and Shi J: miR-33a is up-regulated in chemoresistant osteosarcoma and promotes osteosarcoma cell resistance to cisplatin by down-regulating TWIST. J Exp Clin Cancer Res 33: 12, 2014.

31. Pan W, Wang H, Jianwei R and Ye Z: MicroRNA-27a promotes proliferation, migration and invasion by targeting $M A P 2 K 4$ in human osteosarcoma cells. Cell Physiol Biochem 33: 402-412, 2014.

32. Singh R and Saini N: Downregulation of BCL2 by miRNAs augments drug-induced apoptosis - a combined computational and experimental approach. J Cell Sci 125: 1568-1578, 2012

33. Cimmino A, Calin GA, Fabbri M, Iorio MV, Ferracin M, Shimizu M, et al: miR-15 and miR-16 induce apoptosis by targeting BCL2. Proc Natl Acad Sci USA 102: 13944-13949, 2005.

34. Klein MJ and Siegal GP: Osteosarcoma: Anatomic and histologic variants. Am J Clin Pathol 125: 555-581, 2006. 